\title{
Dynamic Performance of Estimator-based Speed Sensorless Control of Induction Machines Using Extended and Unscented Kalman Filters
}

Research Article

\author{
Krisztián Horváth¹, Márton Kuslits²
}

'Széchenyi István University H-9026 Györ, Egyetem tér 1., Hungary

2Independent Reasercher

Received August 30, 2017; Accepted March 21, 2018

\begin{abstract}
This paper presents an estimator-based speed sensorless field-oriented control (FOC) method for induction machines, where the state estimator is based on a self-contained, non-linear model. This model characterises both the electrical and the mechanical behaviours of the machine and describes them with seven state variables. The state variables are estimated from the measured stator currents and from the known stator voltages by using an estimator algorithm. An important aspect is that one of the state variables is the load torque and, hence, it is also estimated by the estimator. Using this feature, the applied estimator-based speed sensorless control algorithm may be operated adequately besides varying load torque. In this work, two different variants of the control algorithm are developed based on the extended and the unscented Kalman filters (EKF, UKF) as state estimators. The dynamic performance of these variants is tested and compared using experiments and simulations. Results show that the variants have comparable performance in general, but the UKF-based control provides better performance if a stochastically varying load disturbance is present.

Keywords: Induction machine $\bullet$ speed sensorless control • field-oriented control (FOC) • non-linear state estimation • Ioad torque estimation • extended Kalman filter (EKF) • unscented Kalman filter (UKF)
\end{abstract}

\section{Introduction}

Induction machines are widely used in many industrial and research applications. These machines possess wellknown advantages, such as reliability, robustness and affordability. In order to fulfil the performance requirements, induction machine drives need to be controlled. The most commonly used control methods for induction machines are field-oriented control (FOC) and direct torque control (DTC), as discussed and compared by Orłowska-Kowalska and Dybkowski (2016) and Casadei et al. (2002). The two main types of FOC schemes are indirect field-oriented control (IFOC) and direct field-oriented control (DFOC). These FOC structures are discussed by Liu et al. (2015) and De Pelegrin et al. (2016). A possible variant of the DFOC algorithm is the direct rotor field-oriented control (DRFOC), where the applied reference frame is fixed to the rotor flux. In addition to FOC structures in induction machine control, different DTC schemes are also used, e.g. direct self-control (DSC) and direct torque controlspace vector modulation (DTC-SVM), as discussed by Orłowska-Kowalska and Dybkowski (2016). Using FOC or DTC methods, the electromagnetic torque and flux of the machine can be controlled independently.

Usually, these control techniques obtain the speed and position information from a rotary sensor, such as a resolver or an encoder. These sensors, however, might be expensive parts and can be error prone if used under harsh operating conditions. Therefore, elimination of these sensors has many advantages: for instance, reduced cost, increased reliability and smaller size can be achieved. In order to eliminate the rotary sensors in induction 
machine control, the so-called speed sensorless control methods have been developed. Usually, speed sensorless control solutions may rely on signal injection, on deterministic observers or on stochastic estimators, as described by Holtz (2006).

In estimator-based methods, the performance in terms of control depends on the applied estimator type. The most widely used estimator type is the regular linear Kalman filter, which is described by Kalman (1960), Auger et al. (2013) and Šlapák et al. (2016b). In the case of induction machines, non-linear state estimators are usually needed due to the non-linear behaviour of machine models and control algorithms. In Kim et al. (1994) and Leite et al. (2004), application of the extended Kalman filter (EKF) is discussed for a speed sensorless FOC algorithm. EKF is a possible extension of the regular linear Kalman filter for non-linear estimation and based on Taylor series expansion, as described by Šlapák et al. (2016a). In addition to other techniques, EKF is also applied in speed sensorless control of an induction machine in the study by Fodor and Tóth (2004).

An alternative method for non-linear Kalman filtering is the unscented Kalman filter (UKF), which applies the unscented transformation (UT) in the prediction step of a regular Kalman filter, as described by Julier and Uhlmann (1997) and Julier et al. (2000). The principle of UKF generalises the Kalman filter without linearisation for non-linear systems, as introduced in the paper by Julier and Uhlmann (1997). The behaviour of induction machines may be described by strongly non-linear models with sufficient accuracy; therefore, adequate estimation performance can be provided by non-linear UKF, in contrast to the linearisation-based observers or estimators. Works by Rigatos and Siano (2012), Akin et al. (2006) and Jafarzadeh et al. (2012) focus on the state estimation of induction machines using UKF, and these works also provide a comparative analysis between EKF and UKF. In another work (Kumar et al., 2011), three different state estimation methods are discussed, such as EKF, UKF and the neural state filter. Lešić et al. (2012) apply the dual UKF for the state and parameter estimation of induction machines.

Various types of UKF estimators can be applied in induction machine control systems. The architecture of the estimators depends mainly on the selected state variables and the corresponding model equations. Rigatos and Siano (2012) present a six-variable dynamic model of the induction machine for use in the UKF design. The state variables are the rotor mechanical position and speed, the direct $(d)$ component of the rotor flux, $d$ and quadrature ( $q$ ) components of the stator current and the rotor flux angle. Lešić et al. (2012) use the $d$ and $q$ components of the stator current, the magnetising current and the rotor flux angle in the state vector for estimation. In addition, the estimated values are applied in a rotor flux-oriented FOC algorithm as well. In the work of Kumar et al. (2011), two components of stator current, two components of rotor flux and the mechanical speed of the rotor are estimated by UKF. Yildiz et al. (2016) apply a seventh-order induction machine model with UKF estimator in a speed sensorless drive, where the estimated state variables are the stator current and rotor flux components, the rotor mechanical speed, the load torque and the rotor resistance. A similar work by Yildiz et al. (2017) uses the stator resistance as the seventh state variable. In the study by Akin et al. (2006), the electrical rotor speed is used, in addition to the stator current and rotor flux components, for estimation in an IFOC algorithm. Jafarzadeh et al. (2012, 2013) use six state variables, namely, stator flux components, stator current components, electrical speed of rotor and load torque, for estimation in a DTC-based speed control algorithm.

This paper is based on a former conference paper (Horváth and Kuslits, 2017) and presents an estimator-based speed sensorless DRFOC solution, where the estimator is based on a seventh-order machine model. The applied state variables are the $d$ and $q$ components of the stator currents, the magnetising current, the electrical position and speed of the rotor, position of the rotor flux and the load torque. These state variables are selected systematically and, as a novelty, all of the electrical and mechanical quantities result directly from this model considering the internal friction of the machine as well, just contrary to the preceding works (Akin et al., 2006; Jafarzadeh et al., 2012, 2013; Kumar et al., 2011; Lešić et al., 2012; Rigatos and Siano, 2012; Yildiz et al., 2016, 2017). The applied state space model is defined in the rotor flux frame in order to perform non-linear state estimation in a speed sensorless DRFOC structure, which is also presented. Two different variants of this speed sensorless DRFOC algorithm is developed using UKF and, in addition to the concept in Horváth and Kuslits (2017), EKF as state estimators. As a further novelty relative to Horváth and Kuslits (2017), a comparative performance analysis of these variants is carried out by experiments and simulations. In addition to the regular step response analysis, stochastically varying load torque is also applied in order to investigate the load estimation and load disturbance rejection performances of the EKF- and UKF-based variants.

The paper is organised as follows. In Section 2, the fundamental squirrel-cage induction machine model is described, which is applied for control algorithm and estimator design, as well as for simulations. Section 3 describes the EKF and UKF algorithms that are used for state estimation in this paper. Section 4 presents the estimator-based 
speed sensorless DRFOC algorithm. Section 5 describes an implementation example, as well as the experimental and simulation results. Finally, conclusions are drawn in Section 6.

\section{Dynamic model of induction machines}

In this section, the well-known dynamic model of induction machines is described in a rotating arbitrary reference frame, as discussed by Amezquita-Brooks et al. (2015). Using this lumped parameter-type model, an extended seventh-order model of induction machines is introduced in the second part of this section. The applied seventhorder model is a state space model wherein the reference frame is fixed to the rotor flux. By applying the discrete form of the state transition equation in a state estimator algorithm such as EKF or UKF, an estimator-based speed sensorless DRFOC algorithm may be developed.

\subsection{Model of induction machines in general rotating reference frame}

The dynamic model of squirrel-cage induction machines consists of an electrical part and a mechanical part. The electrical part of the model is written in an arbitrarily chosen two-phase rotating reference frame, where $\omega$ is the angular speed of the frame. The two-phase stator voltage equations in this frame are as follows:

$$
v_{d s}=R_{s} i_{d s}+\frac{\mathrm{d}}{\mathrm{dt}} \psi_{d s}-\omega \psi_{q s}
$$

and

$$
v_{q s}=R_{s} i_{q s}+\frac{\mathrm{d}}{\mathrm{dt}} \psi_{q s}+\omega \psi_{d s},
$$

where $v_{d s}, v_{q s}, i_{d s}, i_{q s}, \psi_{d s}$ and $\psi_{q s}$ are the $d$ and $q$ components of the stator voltage, the stator current and the stator flux, and $R_{s}$ is the stator resistance.

Similarly, rotor voltage equations may be written as

$$
v_{d r}=R_{r} i_{d r}+\frac{\mathrm{d}}{\mathrm{dt}} \psi_{d r}-\left(\omega-\omega_{r}\right) \psi_{q r}
$$

and

$$
v_{q r}=R_{r} i_{q r}+\frac{\mathrm{d}}{\mathrm{dt}} \psi_{q r}+\left(\omega-\omega_{r}\right) \psi_{d r},
$$

where $v_{d r}, v_{q r}, i_{d r}, i_{q r}, \psi_{d r}$ and $\psi_{q r}$ are the $d$ and $q$ components of the rotor voltage, the rotor current and the rotor flux, analogously to the stator quantities. $R_{r}$ is the rotor resistance, and $\omega_{r}$ is the rotor electrical speed. In squirrel-cage induction machines, the rotor electrical circuit is short-circuited, and therefore, conditions $v_{d r}=0 \mathrm{~V}$ and $v_{q r}=0 \mathrm{~V}$ are used afterwards.

The stator and rotor flux equations in Eqs. (1)-(4) may be expressed as follows:

$$
\begin{aligned}
& \psi_{d s}=L_{s} i_{d s}+L_{m} i_{d r}, \\
& \Psi_{q s}=L_{s} i_{q s}+L_{m} i_{q r}, \\
& \psi_{d r}=L_{r} i_{d r}+L_{m} i_{d s}
\end{aligned}
$$

and

$$
\psi_{q r}=L_{r} i_{q r}+L_{m} i_{q s}
$$

where $L_{s}$ and $L_{m}$ are the stator and mutual inductances and $L_{r}$ is the rotor inductance. 
The electromagnetic torque $T_{e}$ follows from the stator current and rotor flux components:

$$
T_{e}=\frac{3}{2} N \frac{L_{m}}{L_{r}}\left(i_{q s} \psi_{d r}-i_{d s} \psi_{q r}\right)
$$

where $N$ is the number of pole pairs.

The equation of motion represents the mechanical behaviour of the model:

$$
\frac{\mathrm{d}}{\mathrm{dt}} \omega_{m}=\frac{T_{e}-D \omega_{m}-T_{0} \operatorname{sgn}\left(\omega_{m}\right)-T_{\text {load }}}{\theta}
$$

where $\omega_{m}$ is the rotor mechanical speed, $D$ is the viscous friction coefficient, $T_{0}$ is the static friction, $T_{\text {load }}$ is the external load torque and $\theta$ is the total inertia. The rotor mechanical speed may be calculated from the rotor electrical speed, dividing it by the number of pole pairs: $\omega_{m}=\frac{1}{N} \omega_{r}$.

\subsection{Rotor flux-oriented state-space model of induction machines}

Eqs. (1)-(10) describe the dynamic behaviour of the induction machines in any rotating reference frame, where $\omega$ is the rotational speed of the frame. In this case, the applied reference frame is fixed to the rotor flux. From this, it follows that $\psi_{d r}$ is varying but the $q$ component of the rotor flux results as $\psi_{q r}=0 \mathrm{~Wb}$. As a further result, Eqs. (3), (4), (8) and (9) may be written in a simpler form in the applied frame. In order to simplify the forthcoming expressions, let us define the magnetising current as

$$
i_{m r}=\frac{\psi_{d r}}{L_{m}}
$$

Further, $i_{m r}$ can be calculated from $i_{d s}$ as $I_{m r}(s)=G(s) I_{d s}(s)$ where the transfer function $G(s)$ is

$$
G(s)=\frac{I_{m r}(s)}{I_{d s}(s)}=\frac{1}{T_{r} s+1},
$$
in which $T_{r}=\frac{L_{r}}{R_{r}}$ is the rotor time constant. Using $i_{m r}$, the position of the rotor flux can be calculated by the following
expression:

$$
\varphi_{e}=\int \omega_{r}+\frac{R_{r}}{L_{r}} \frac{i_{q s}}{i_{m r}} \mathrm{dt}
$$

In order to simplify the expressions, let us define the leakage inductance as

$$
\sigma=1-\frac{L_{m}^{2}}{L_{s} L_{r}}
$$

Using Eqs. (1)-(14), the non-linear state transition equation of squirrel-cage induction machines may be written. In order to apply the state transition equation for estimator design in the speed sensorless DRFOC algorithm, let us define the input vector of the system as $u=\left[v_{d s} v_{q s}\right]^{T}$ and the measurement vector as $z=\left[i_{d s} i_{q s}\right]^{T}$. For the detailed, self-containing description of the induction machines, a full-order model is necessary, which contains four electrical state variables and two mechanical state variables at least. The mechanical state variables are the electrical speed $\left(\omega_{r}\right)$ and position of the rotor $\left(\varphi_{r}\right)$, where $\varphi_{r}=\int \omega_{r} \mathrm{dt}$. The proposed state transition equation may be used in the EKF algorithm only with continuous functions. Thus, in the state transition equation, the sign function of Eq. (10) is approximated by a sigmoid function as $\operatorname{sgn}\left(\omega_{m}\right) \approx \frac{\omega_{m}}{\sqrt{1+\omega_{m}^{2}}}$. For the electrical state variables, the $d$ and $q$ components 
of the stator currents, the magnetising current $\left(i_{m r}\right)$ and position of rotor flux $\left(\varphi_{e}\right)$ are selected. The components of the stator currents are necessary for control of both the flux of the machine and the electromagnetic torque. The magnetising current is related to the magnitude of rotor flux, and the position of rotor flux is required for coordinate transformations. In order to estimate the external load torque, let us extend the full-order state space model with the load torque as an additional state variable. By using this extension, the state vector may be defined as $x=\left[i_{d s} i_{q s} i_{m r}\right.$ $\left.\varphi_{r} \omega_{r} \varphi_{e} T_{\text {load }}\right]^{T}$ and the state transition equation in continuous time may be written as follows:

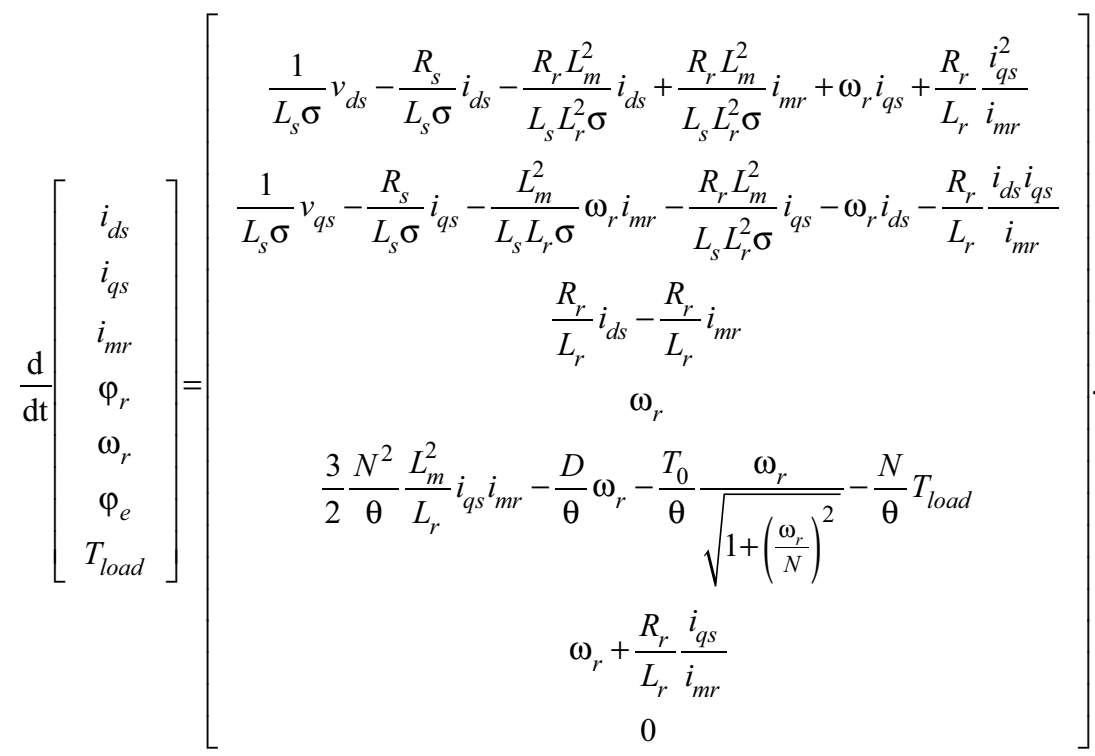

Regarding Eq. (18), the measurement matrix of the model can be written as

$$
H=\left[\begin{array}{lllllll}
1 & 0 & 0 & 0 & 0 & 0 & 0 \\
0 & 1 & 0 & 0 & 0 & 0 & 0
\end{array}\right]
$$

An important limitation of this model is that it is supposed that all of the model parameters are time-invariant constants. In other words, possible parameter variations, e.g. change of the stator resistance as a function of temperature, are not considered.

\section{The EKF and the UKF state estimator algorithms}

In this section, the conventional EKF and the more advanced UKF algorithms are presented. First, the characteristic properties and applicability of the EKF and UKF algorithms are discussed. Second, the general equations shared by both algorithms are described. Finally, the EKF and UKF algorithms are introduced.

\subsection{Characteristics and applicability of the algorithms}

Estimation of state variables may be a difficult problem if the observed plant is a non-linear system and the noise is stochastic. The simplest way to approach this problem is to linearise the plant model as in the EKF. This approximation provides good dynamic behaviour in many applications but may lead to inaccurate results, as mentioned by Jafarzadeh et al. (2012). This follows from the assumption that all of the transformations are quasilinear. However, the linearisation-based observation and estimation methods suffer a serious limitation due to this assumption, because the linearised transformations are only reliable if the error propagation is well approximated by a linear function as described in the study by Julier and UhImann (2004). The approximation might be extremely poor if this assumption is not true.

A further important question is the applicability of the algorithms. Implementation requires similar efforts in both cases since the EKF and the UKF rely on the same non-linear state transition equation and the noise covariance 
parameters are treated similarly as well. Regarding the computational burdens, however, it is clear that the UKF requires significantly more real-time calculations due to the UT. Nevertheless, this is not an actual problem nowadays because state-of-the-art high-performance multicore microcontrollers with floating point arithmetic, field programmable gate array (FPGA), system on chip (SoC) and application-specific integrated circuit (ASIC) technologies may provide the necessary computing performance for the practical applications of the UKF-based solutions.

\subsection{General equations}

In order to derive the EKF and UKF algorithms for state estimation, the state transition equation and the measurement model have to be defined. The discrete non-linear state transition equation may be written as

$$
x_{k+1}=\tilde{f}\left(x_{k}, u_{k}\right)+w_{k}
$$

where $x_{k}$ is the state vector, $u_{k}$ is the input vector, $w_{k}$ is the process noise at time $k$ and $\tilde{f}\left(x_{k}, u_{k}\right)$ is a given non-linear function. The measurement model in most of the practical applications might be linear and can be written as follows:

$$
z_{k}=H x_{k}+v_{k}
$$

where $z_{k}$ is the measurement vector, $v_{k}$ is the measurement noise at time $k$ and $H$ is the measurement matrix. In Eqs. (17) and (18), the elements $w_{k}$ and $v_{k}$ are probabilistic variables. Realisations of $w_{k}$ and $v_{k}$ in time produce realisations of white noise processes with zero means and with $Q$ and $R$ covariance matrices, as described by Akin et al. (2006) and Jafarzadeh et al. (2012).

\subsection{The EKF algorithm}

Similarly to the regular Kalman filter, the EKF is an iterative algorithm and consists of a prediction step, which is followed by a correction step. First, the predicted state $\hat{x}_{k+1}^{-}$can be calculated using the given non-linear function as follows:

$$
\hat{x}_{k+1}^{-}=\tilde{f}\left(\hat{x}_{k}^{+}, u_{k}\right)
$$

where $\hat{x}_{k}^{+}$is the corrected state vector. At the end of the prediction step, the predicted error covariance matrix $P_{k+1}^{-}$is calculated by the following expression:

$$
P_{k+1}^{-}=F_{k} P_{k}^{+} F_{k}^{T}+Q
$$

where $P_{k}^{+}$is the corrected error covariance matrix. $F_{k}$ is the Jacobian of the given non-linear expression and it is defined as follows:

$$
F_{k}=\left.\frac{\partial \tilde{f}(x, u)}{\partial x}\right|_{x=\hat{x}_{k}^{+}, u=u_{k}}
$$

In the correction step, equations of the regular discrete Kalman filter may be applied as follows:

$$
\begin{aligned}
& K_{k+1}=P_{k+1}^{-} H^{T}\left(H P_{k+1}^{-} H^{T}+R\right)^{-1} \\
& \hat{x}_{k+1}^{+}=\hat{x}_{k+1}^{-}+K_{k+1}\left(z_{k+1}-H \hat{x}_{k+1}^{-}\right) \\
& P_{k+1}^{+}=\left(I-K_{k+1} H\right) P_{k+1}^{-}
\end{aligned}
$$

where $K_{k+1}$ is the Kalman gain at time $k+1$. 


\subsection{The UKF algorithm}

Similarly to the EKF, UKF is also an iterative algorithm; however, it applies more complex iterations. In the prediction step, the UKF applies the UT in order to select a set of sigma points $\sigma_{\hat{x}_{k}^{+}}^{(i)}$ with the same mean $\hat{x}_{k}^{+}$and covariance $P_{k}^{+}$as the state vector $x_{k}$. Similarly to Jafarzadeh et al. (2012) and Biswas et al. (2017), the general UT is applied in this work, which means that the number of sigma points is $2 n+1$, where $n$ is the number of state variables. For general UT, the sigma points can be defined as follows:

$$
\sigma_{\hat{x}_{k}^{+}}^{(i)}=\left\{\begin{array}{lc}
\hat{x}_{k}^{+} & \text {for } i=0 \\
\hat{x}_{k}^{+}+\left(\sqrt{(n+\kappa) P_{k}^{+}}\right)_{(i)}^{T} & \text { for } i=1, \ldots, n \\
\hat{x}_{k}^{+}-\left(\sqrt{(n+\kappa) P_{k}^{+}}\right)_{(i-n)}^{T} & \text { for } i=n+1, \ldots, 2 n
\end{array}\right.
$$

where $\left(\sqrt{(n+\kappa) P_{k}^{+}}\right)_{(i)}^{T}$ is the $i^{\text {th }}$ row of matrix $\left(\sqrt{(n+\kappa) P_{k}^{+}}\right)^{T}$, and $\kappa$ is a design parameter. In Eq. (25), the term $\sqrt{(n+\kappa) P_{k}^{+}}$is the Cholesky factorisation of matrix $(n+\kappa) P_{k}^{+}$, as described in the studies by Jafarzadeh et al. (2012) and Lešić et al. (2012).

Sigma points have weights that depend on the design parameter $\kappa$, according to the following expression:

$$
W^{(i)}=\left\{\begin{array}{cc}
\frac{\kappa}{n+\kappa} & \text { for } i=0 \\
\frac{1}{2(n+\kappa)} & \text { for } i=1, \ldots, 2 n
\end{array}\right.
$$

The projected sigma points can be calculated by using the known non-linear transformation (17) as follows:

$$
\sigma_{\hat{x}_{k+1}^{-}}^{(i)}=\tilde{f}\left(\sigma_{\hat{x}_{k}^{+}}^{(i)}, u_{k}\right) \text {. }
$$

The predicted state $\hat{x}_{k+1}^{-}$may be calculated from Eqs. (26)-(27):

$$
\hat{x}_{k+1}^{-}=\sum_{i=0}^{2 n} W^{(i)} \sigma_{\hat{x}_{k+1}^{-}}^{(i)}
$$

At the end of the prediction step, the predicted error covariance matrix $P_{k+1}^{-}$is calculated by the following equation:

$$
P_{k+1}^{-}=\sum_{i=0}^{2 n}\left[W^{(i)}\left(\sigma_{\hat{x}_{k+1}^{-}}^{(i)}-\hat{x}_{k+1}^{-}\right)\left(\sigma_{\hat{x}_{k+1}^{-}}^{(i)}-\hat{x}_{k+1}^{-}\right)^{T}\right]+Q .
$$

In the correction step of the UKF algorithm, Eqs. (22)-(24) of the discrete Kalman filter might be applied.

\section{The applied speed sensorless control algorithm}

In this section, the applied speed sensorless DRFOC algorithm is presented. This algorithm is used for the control of the electromagnetic torque and flux of a squirrel-cage induction machine, as depicted in Figure 1. The described DRFOC architecture is suitable to develop an estimator-based speed sensorless control algorithm. 
The estimator of the control algorithm has been implemented by using the EKF and UKF algorithms, which are described in Section 3.

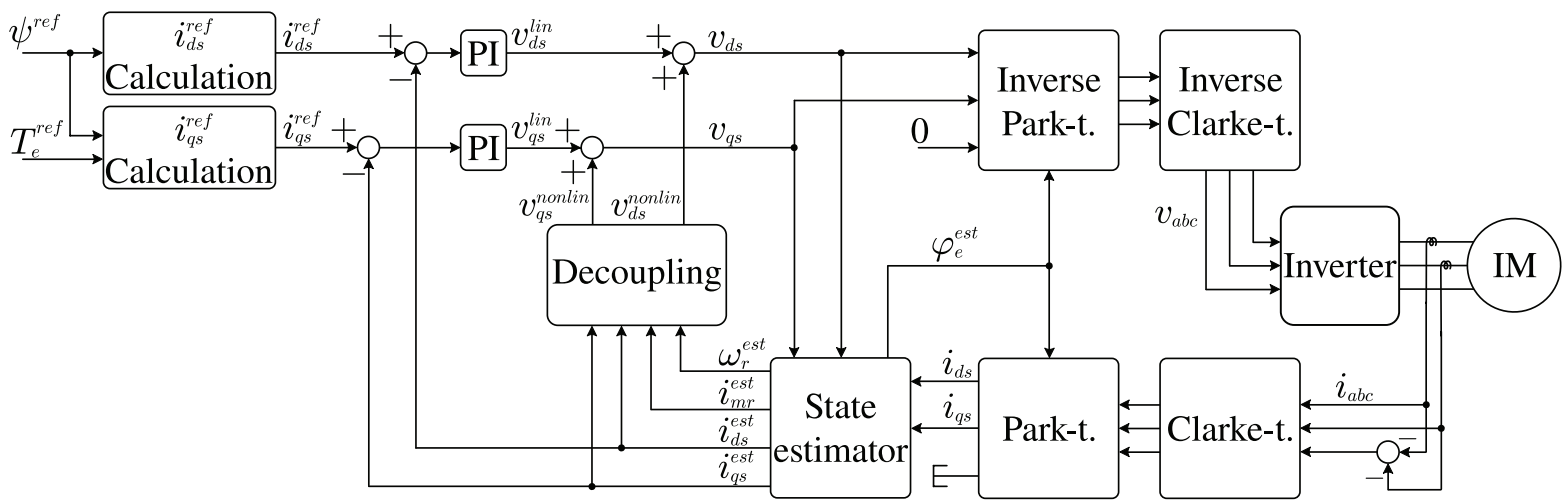

Fig. 1. Block diagram of the estimator-based speed sensorless DRFOC algorithm

In the applied control scheme, the $i_{a}$ and $i_{b}$ phase currents are measured, and the $i_{c}$ phase current is calculated according to Kirchhoff's law for Y-circuit $\left(i_{a}+i_{b}+i_{c}=0 \mathrm{~A}\right)$. From the phase currents, the $i_{d s}$ and $i_{q s}$ stator current components are calculated with the Clarke and Park transformations. The applied form of the Clarke and Park transformation matrices are as follows:

$$
C=\frac{2}{3}\left[\begin{array}{ccc}
1 & -\frac{1}{2} & -\frac{1}{2} \\
0 & \frac{\sqrt{3}}{2} & -\frac{\sqrt{3}}{2} \\
\frac{1}{2} & \frac{1}{2} & \frac{1}{2}
\end{array}\right] \text { and } P=\left[\begin{array}{ccc}
\cos \varphi_{e} & \sin \varphi_{e} & 0 \\
-\sin \varphi_{e} & \cos \varphi_{e} & 0 \\
0 & 0 & 1
\end{array}\right]
$$

The position of the rotor flux is estimated by the estimator and applied for Park transformation, as can be seen in Figure 1. Apart from the rotor flux position, the estimator estimates the $d$ and $q$ components of the stator current, the magnetising current, the position and speed of the rotor as well as the load torque. Input variables of the estimator are the stator currents and the known stator voltages, which are interpreted in the applied rotating reference frame that is aligned to the rotor flux. However, the estimator algorithm is written in its discrete form, and thus, the nonlinear state transition equation must be written in discrete form too, similarly to (17). The discrete form of Eq. (15) may be written as follows:

$$
x_{k+1}=f\left(x_{k}, u_{k}\right) T_{s}+x_{k}
$$

where $T_{s}$ is the sampling time of the discrete system.

The estimated $d$ and $q$ components of the stator current are used for feedback. Through the $d$ and $q$ components of the stator current, the flux of the machine and the electromagnetic torque, respectively, can be controlled.

The current references for the DRFOC algorithm can be calculated by the following equations:

$$
i_{d s}^{r e f}=\frac{\psi^{r e f}}{L_{m}} \text { and } i_{q s}^{r e f}=\frac{2}{3} \frac{1}{N} \frac{L_{r}}{L_{m}} \frac{T_{e}^{r e f}}{\psi^{r e f}}
$$

where $\psi^{\text {ref }}$ is the flux reference and $T_{e}^{r e f}$ is the electromagnetic torque reference.

One can see that Eqs. (1) and (2) are cross-coupled through Eqs. (5) and (6). This is an adverse property of the machine model regarding it from the control design point of view. In order to mitigate this problem, an additional decoupling algorithm is needed. For the derivation of this algorithm, let us rewrite the $d$-axis stator voltage equation as follows: 


$$
v_{d s}=\underbrace{\left[R_{s} i_{d s}+L_{s} \sigma \frac{\mathrm{d}}{\mathrm{dt}} i_{d s}\right]}_{v_{d s}^{\text {lin }}}+\underbrace{\left[\frac{L_{m}^{2}}{L_{r}} \frac{\mathrm{d}}{\mathrm{dt}} i_{m r}-L_{s} \sigma\left(\omega_{r}+\frac{R_{r}}{L_{r}} \frac{i_{q s}}{i_{m r}}\right) i_{q s}\right]}_{v_{d s}^{\text {nonlin }}}
$$

where $v_{d s}^{\text {lin }}$ is the linear part, and $v_{d s}^{\text {nonlin }}$ is the non-linear part of the equation. The same problem applies to the $q$-axis stator voltage equation, which can be split similarly:

$$
v_{q s}=\underbrace{\left[R_{s} i_{q s}+L_{s} \sigma \frac{\mathrm{d}}{\mathrm{dt}} i_{q s}\right]}_{v_{q s}^{\text {lin }}}+\underbrace{\left[\left(\omega_{r}+\frac{R_{r}}{L_{r}} \frac{i_{q s}}{i_{m r}}\right)\left(L_{s} \sigma i_{d s}+\frac{L_{m}^{2}}{L_{r}} i_{m r}\right)\right]}_{v_{q s}^{\text {nonlin }}}
$$

where $v_{q s}^{l i n}$ is the linear part, and $v_{q s}^{\text {nonlin }}$ is the non-linear part of the equation. By using the decompositions of Eqs. (33) and (34), current controllers can be designed for the linear part of the plants, and the non-linear parts can be decoupled by an additional algorithm, as can be seen in Figure 1. The decoupling algorithm may be implemented using $v_{d s}^{\text {nonlin }}$ and $v_{q s}^{\text {nonlin }}$, which may be calculated from the estimated stator currents, the magnetising current and the rotor electrical speed.

From $v_{d s}$ and $v_{q s}$, the phase voltages of the machine can be obtained through multiplications by $P^{-1}$ and $C^{-1}$, and the outputs of the inverse Clarke transformation block can be fed as control signals to the inverter.

\section{Experimental and simulation results}

In this section, experimental and simulation results of the estimator-based speed sensorless DRFOC algorithm are presented, where applications of the EKF and UKF algorithms are compared. First, the general conditions and the applied nominal model parameters are described. Afterwards, regular step response experiments and simulations are performed. In the last subsection, results of step response simulations besides stochastically varying load torque are presented.

\subsection{General conditions, parameters and experimental setup}

This subsection describes the general conditions, the parameters applied in the experiments, the simulations and the experimental setup.

For the experiments, a Lenze MCA 10140-RSOB2-Z0C0-STBS00N-R0SU induction machine is applied, which has $0.8 \mathrm{~kW}$ rated power. The nominal parameters of the machine are $N=2, R_{s}=4.7 \Omega, R_{r}=5.2 \Omega, L_{s}=0.1788 \mathrm{H}, L_{r}=0.1790 \mathrm{H}$, $L_{m}=0.1690 \mathrm{H}, \theta=0.00024 \mathrm{kgm}^{2}, D=0.0011 \mathrm{Nms} / \mathrm{rad}, T_{0}=0.075 \mathrm{Nm}$. These are applied in the simulations as well.

The sample time of the control algorithm is $T_{s}=10^{-4} \mathrm{~s}$, and the sample time of the simulation model is $T_{\text {sim }}=$ $10^{-5} \mathrm{~s}$. Design parameters of the EKF and the UKF estimators are $Q_{E K F}=\operatorname{diag}\left\{2.18 \cdot 10^{-3}, 0.78,1.11 \cdot 10^{-5}, 0.15,1.26 \cdot 10^{-3}\right.$, $\left.4.05,3.08 \cdot 10^{-4}\right\}, R_{E K F}=\operatorname{diag}\{78.24,78.24\}$ and $\kappa=1, Q_{U K F}=\operatorname{diag}\left\{1.77 \cdot 10^{-4}, 3.17 \cdot 10^{-2}, 2.49 \cdot 10^{-5}, 0.10,3.44 \cdot 10^{-3}, 2.22 \cdot 10^{-5}\right.$, $\left.5.34 \cdot 10^{-4}\right\}$ and $R_{U K F}=\operatorname{diag}\{5.03,5.03\}$. The noise covariance parameters $Q_{E K F}, R_{E K F}, Q_{U K F}$ and $R_{U K F}$ are determined by exactly the same heuristic procedure, similarly to that described by Zerdali and Barut (2017). The initial value of the state vector is $x_{0}=\left[\begin{array}{llllll}0 & 0 & 0.01 & 0 & 0 & 0\end{array}\right]^{T}$, where $x_{0}^{(3)} \neq 0$ in order to avoid division by zero in Eq. (13). The initial value of the noise covariance matrix is $P_{0}=\operatorname{diag}\left\{10^{-4}, \ldots, 10^{-4}\right\}$. The proportional and integral gains of the current controllers are $P=4.7$ and $I=1148.0$, respectively.

The scheme of the experimental setup may be seen in Figure 2. In order to execute the UKF algorithm in real time, a distributed system is applied based on a high-performance Simulink Real-Time target computer, which is built on a HP BX383AV PC with Intel Core i5-2500 @3.3 GHz CPU. In addition to the execution of the control algorithm, the target computer performs the measurement data acquisition as well. The target computer receives the measured currents from and sends the excitation voltages to the Texas Instruments (TI) TMS570LC437 microcontroller through synchronised communication. The microcontroller performs the measurements and calculates the PulseWidth Modulation (PWM) signals for the TI DRV8301-HC-EVM power stage. The experimental setup is monitored 
by a host personal computer (PC), which is connected to the real-time target by Transmission Control Protocol/ Internet Protocol (TCP/IP) connection.

Host PC (experiment control and data acquisition)
Simulink Real-Time

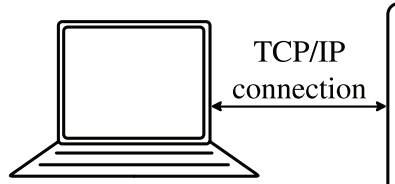
target

Microcontroller

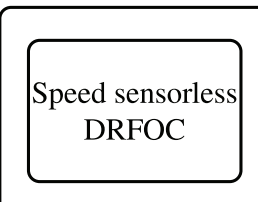

Power electronic board

(Texas Instruments

DRV8301-HC-EVM Rev-D) Induction

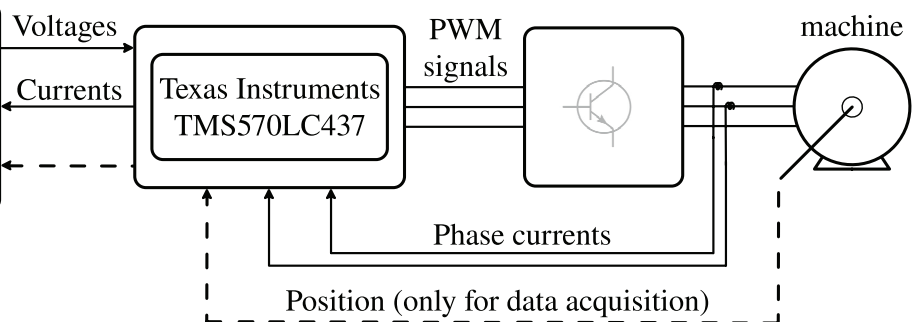

Fig. 2. Experimental setup

\subsection{Step response simulations and experiments}

In this example, step response simulations and experiments are performed with $T_{e}^{\text {ref }}=0.18$ Nm torque and $\psi^{\text {ref }}=$ $0.1 \mathrm{~Wb}$ flux references. Both the EKF and the UKF are used as the state estimator in the control algorithm, and the results are compared.

The results can be seen in Figures 3-5. For the sake of brevity, only the $q$ component of the current is investigated in Figure 3. One may see that the ramp-up of $i_{q s}$ is slightly smoother with the EKF than with the UKF, but both of the algorithms perform adequately during the starting transient. It can also be concluded that the simulation results follow the experimental results as far as can be compared with the noisy measurements. In the steady state, both algorithms work equally well, as can be seen in Figures 4 and 5, which compare the measured and the estimated variables on a longer time span.

(a)
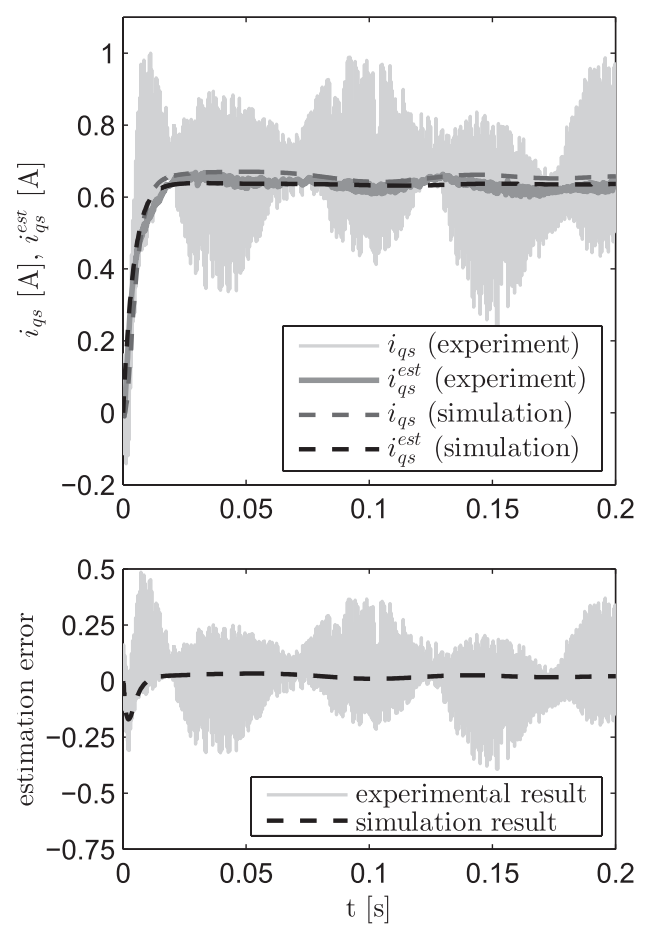

(b)
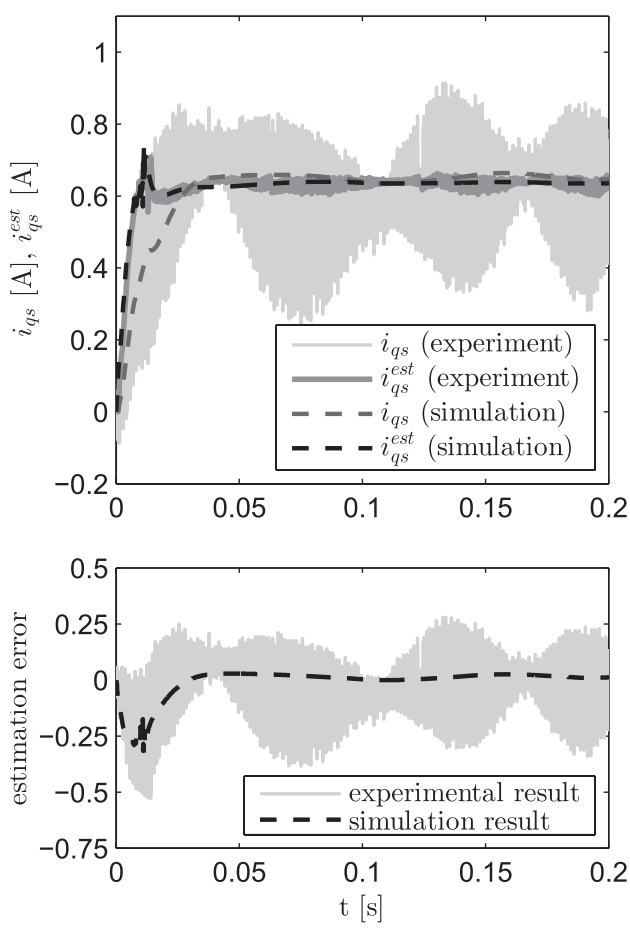

Fig. 3. Comparison of step response simulations and experiments using EKF (a) and UKF (b) 

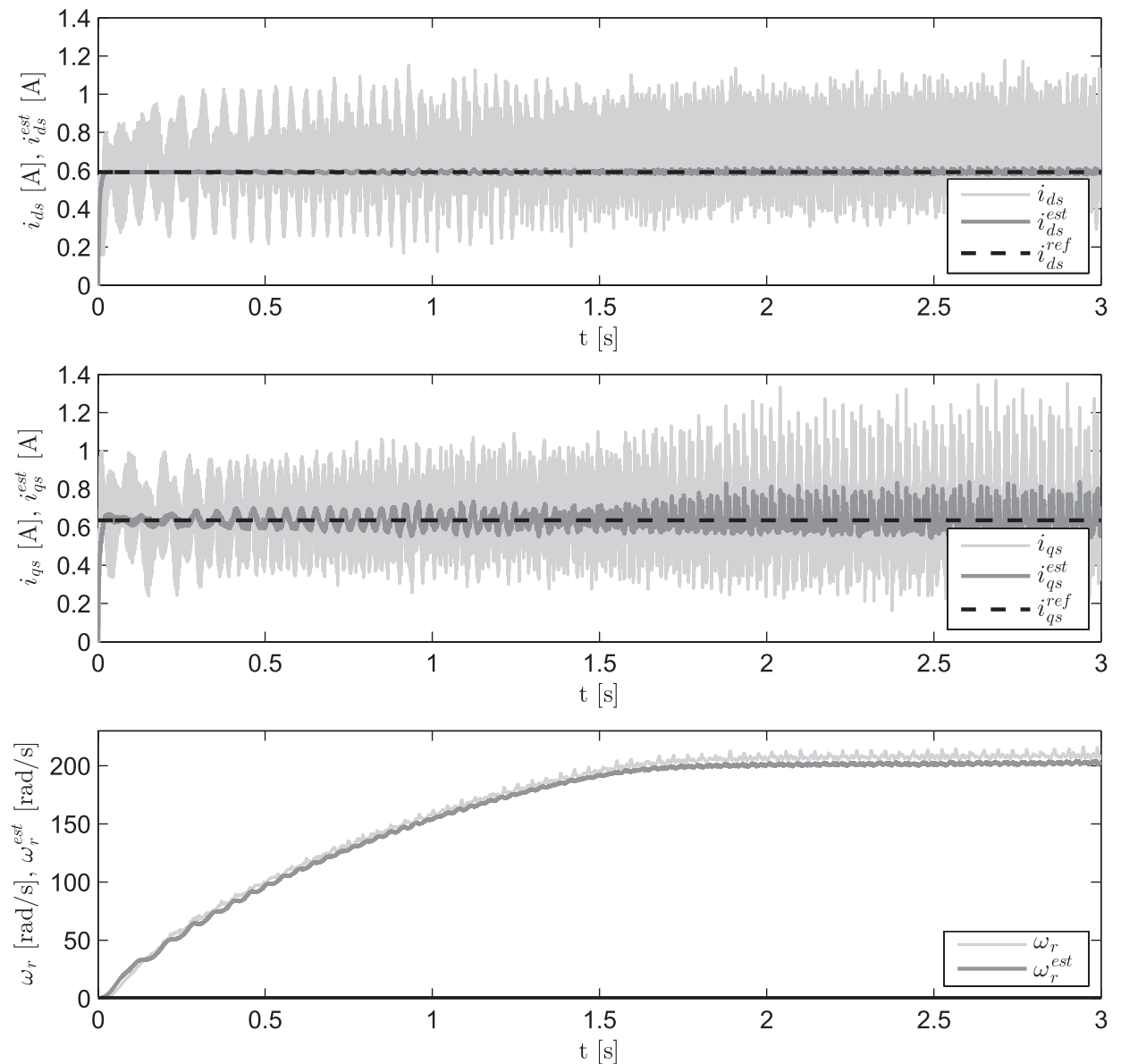

Fig. 4. Comparison of actual and estimated variables (EKF)

\subsection{Step response simulation with stochastically varying load}

In this example, estimation of the load torque and performance of the torque control are investigated besides stochastically varying load torque. This kind of load variations hold significant importance regarding the possible applications of electric drive systems. For example, vehicle drives operating under harsh conditions may face stochastically varying rolling resistance, as mentioned by Vantsevich and Blundell (2015). In order to realistically approximate the stochastically varying load torque, a Wiener process is applied.

For the definition of the Wiener process in this problem, let us define an infinite sequence of independent, identically distributed random variables with normal distribution as

$$
\xi_{1}, \xi_{2}, \ldots, \xi_{\infty}
$$

where $\mathrm{E}\left[\xi_{i}\right]=0$ and $\operatorname{Var}\left(\xi_{i}\right)=\varepsilon, \forall i \in \mathbb{N}$ and where $\mathrm{E}[\cdot]$ and $\operatorname{Var}(\cdot)$ denote the expected value and the variance operators. By using Eq. (35), we may define the stochastic process as follows:

$$
W_{n}(t)=\frac{1}{\sqrt{n}} \sum_{1 \leq i \leq\lfloor n t\rfloor} \xi_{i}
$$

Applying the central limit theorem on Eq. (36), we obtain the following expression:

$$
W(t)=\underset{\substack{n \\ n \rightarrow \infty}}{W}(t)
$$



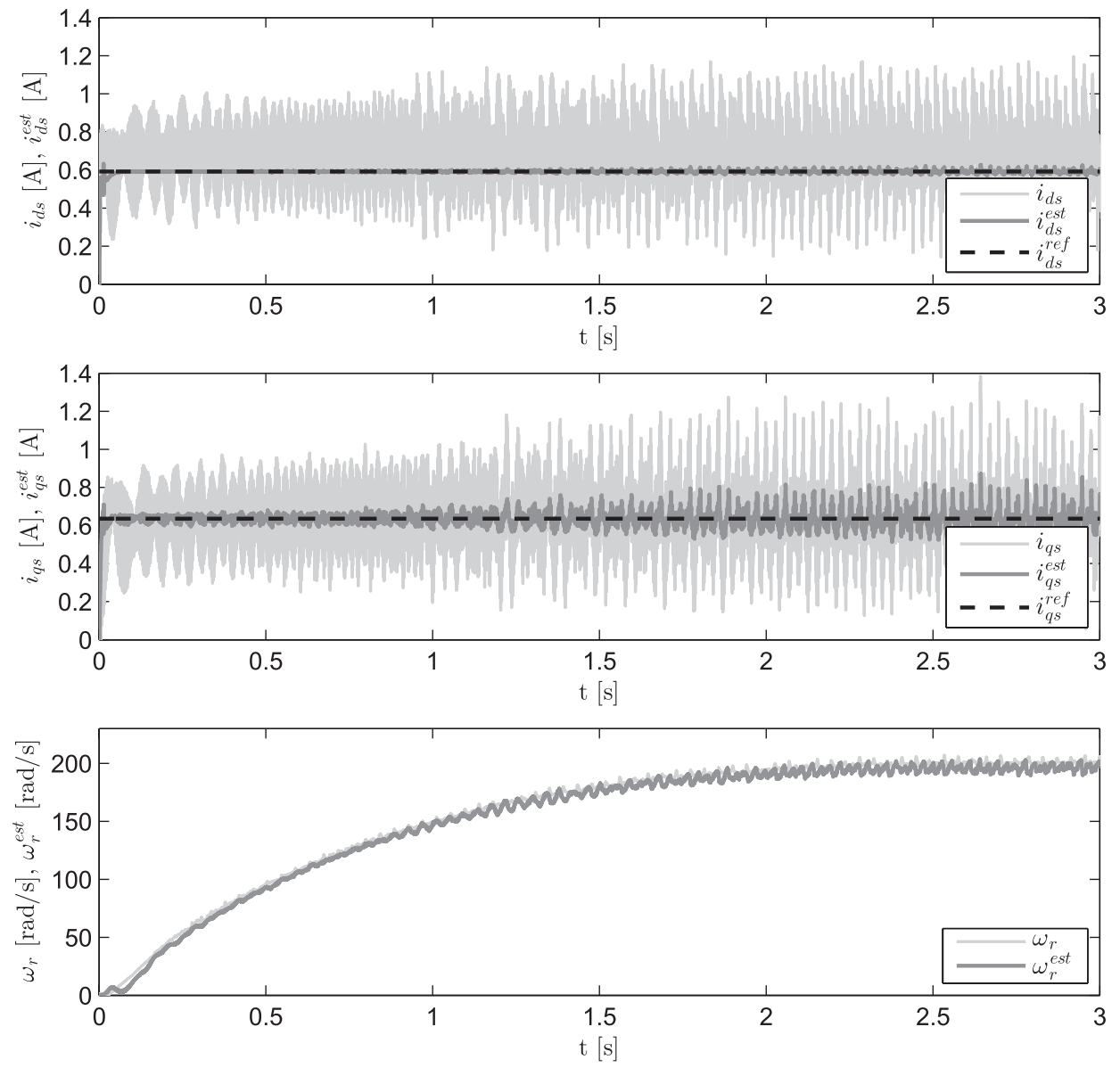

Fig. 5. Comparison of actual and estimated variables (UKF)

which is a Wiener process, as shown by Lalley (2001). However, since the applied numerical simulation method is discretised in time, Eq. (36) is applied in the simulation model as an approximation of Eq. (37) with a reasonably high $n$.

In order to utilise Eq. (36) for load simulation, let us define $w_{n}(t)$ as a realisation of $W_{n}(t)$. By using this definition, a certain realisation of the stochastically varying load torque results as follows:

$$
T_{\text {load }}(t)=T_{\text {load }_{0}}+w_{n}(t)
$$

where $T_{\text {load }_{0}}$ is a static load component. By using (38), various simulations are performed with $T_{\text {load }_{0}}=0 \mathrm{Nm}$, $n=1 / T_{\text {sim }}$ and the variance value $\varepsilon=\left(T_{e}^{r e f} / 2\right)^{2} / t$. This particular value of $\varepsilon$ has been set according to the characteristic properties of the possible random changes in $T_{\text {load }}$ and considering that $T_{\text {load }}$ should not exceed $T_{e}^{\text {ref }}$ beyond a certain probability. The above expression for $\varepsilon$ can be derived as follows. Let us start from the variance of the Wiener process using the approximation Eq. (36):

$$
\operatorname{Var}\left(W_{n}(t)\right)=\operatorname{Var}\left(\frac{1}{\sqrt{n}} \sum_{1 \leq i \leq\lfloor n t\rfloor} \xi_{i}\right)
$$


Considering that $1 / \sqrt{n}$ is a linear transformation in Eq. (39) and that all $\xi_{i}, \forall i \in \mathbb{N}$ are uncorrelated, we can apply the Bienaymé equality according to Loeve (2012, p. 12), by which Eq. (39) might be rewritten as follows:

$$
\operatorname{Var}\left(W_{n}(t)\right)=\frac{1}{n} \sum_{1 \leq i \leq\lfloor n t\rfloor} \operatorname{Var}\left(\xi_{i}\right) \approx \frac{1}{n} n t \operatorname{Var}\left(\xi_{i}\right)=t \varepsilon .
$$

Since $\xi_{i}$ has been defined with normal distribution, it follows that $W_{n}(t)$ is normally distributed as well ${ }^{1}$, with variance $t \varepsilon$. Considering the properties of the normal distribution, let it be required that $T_{\text {load }}$ should not exceed $T_{e}^{\text {ref }}$ besides the probability of $0.9545,{ }^{2}$ which means that the inequality $t \varepsilon \leq\left(T_{e}^{r e f} / 2\right)^{2}$ must be satisfied. From this, the above choice of $\varepsilon$ follows straightforwardly.

Simulation results besides different realisations of the stochastically varying load torque, can be seen in Figures 6 and 7 . Figure 6 shows the estimation of the load torque. It can be seen that after a short overshoot at the beginning, the UKF provides better performance as it follows $T_{\text {load }}$ closer than the EKF.
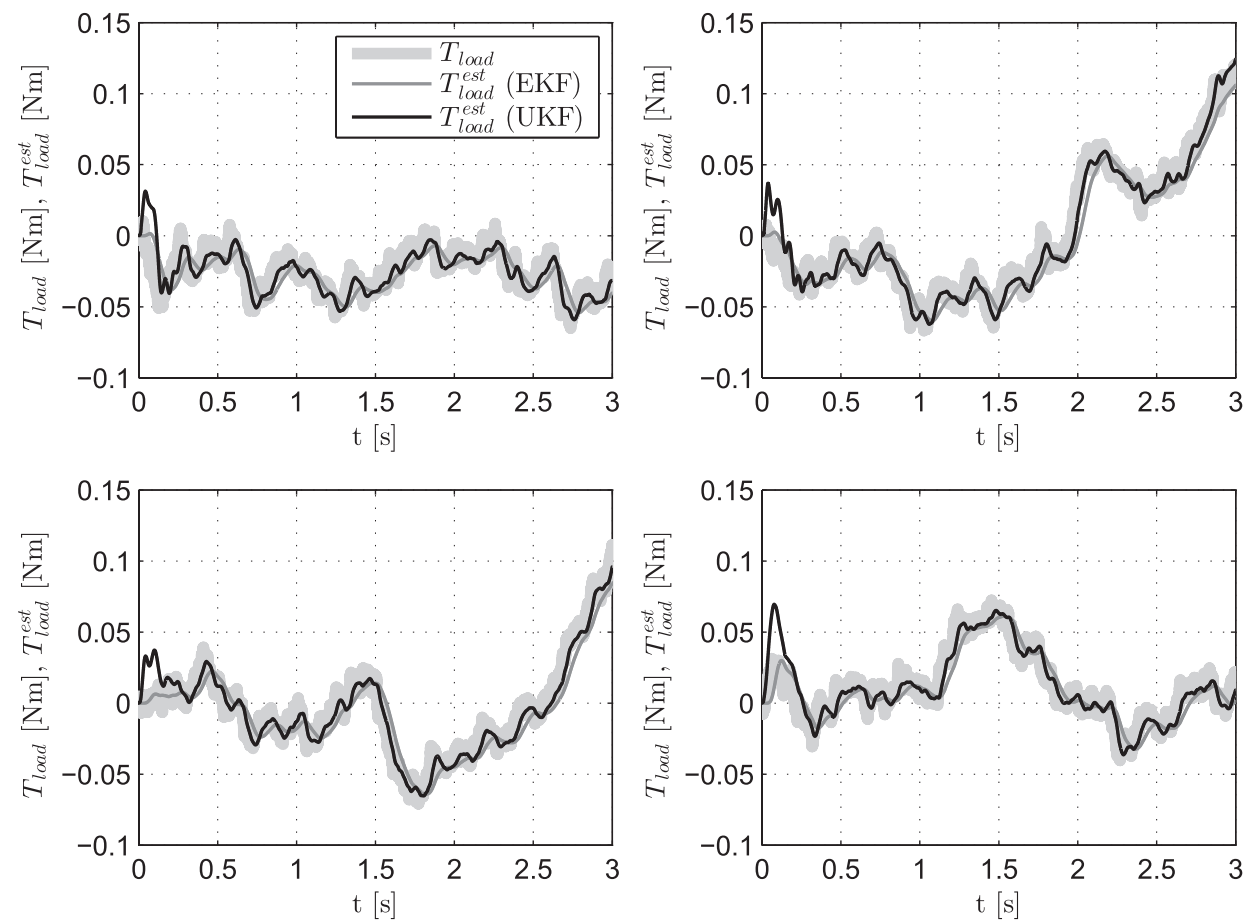

Fig. 6. Load torque estimation besides different realisations of $W_{n}(t)$

Figure 7 shows the performance of the torque controllers. One may conclude that the UKF-based control method provides significantly better performance. In the steady state, the reference torque can be maintained with different errors using EKF and UKF. We may statistically quantify the disturbance rejection capabilities of the control system by introducing the control error $e_{T_{e}}$ and fitting a probability density function to $e_{T_{e}}, t>0.3 \mathrm{~s}$, and calculating the variance of it. One can see that $e_{T_{e}}$ is approximately normally distributed as well and $\operatorname{Var}\left(e_{T_{e}}\right)$ is bounded besides different realisations of the stochastically varying load torque. As can be seen in Table 1, there are significant differences between the variances of the EKF- and the UKF-based results.

1 The sum of independent normally distributed random variables is a normally distributed random variable as well. See Eisenberg and Sullivan (2008).

2 This value results from the basic properties of the normal distribution, also known as the probability corresponding to the four sigma range or as the 68-95-99.7 rule; e.g. see Grafarend (2006, p.553). 

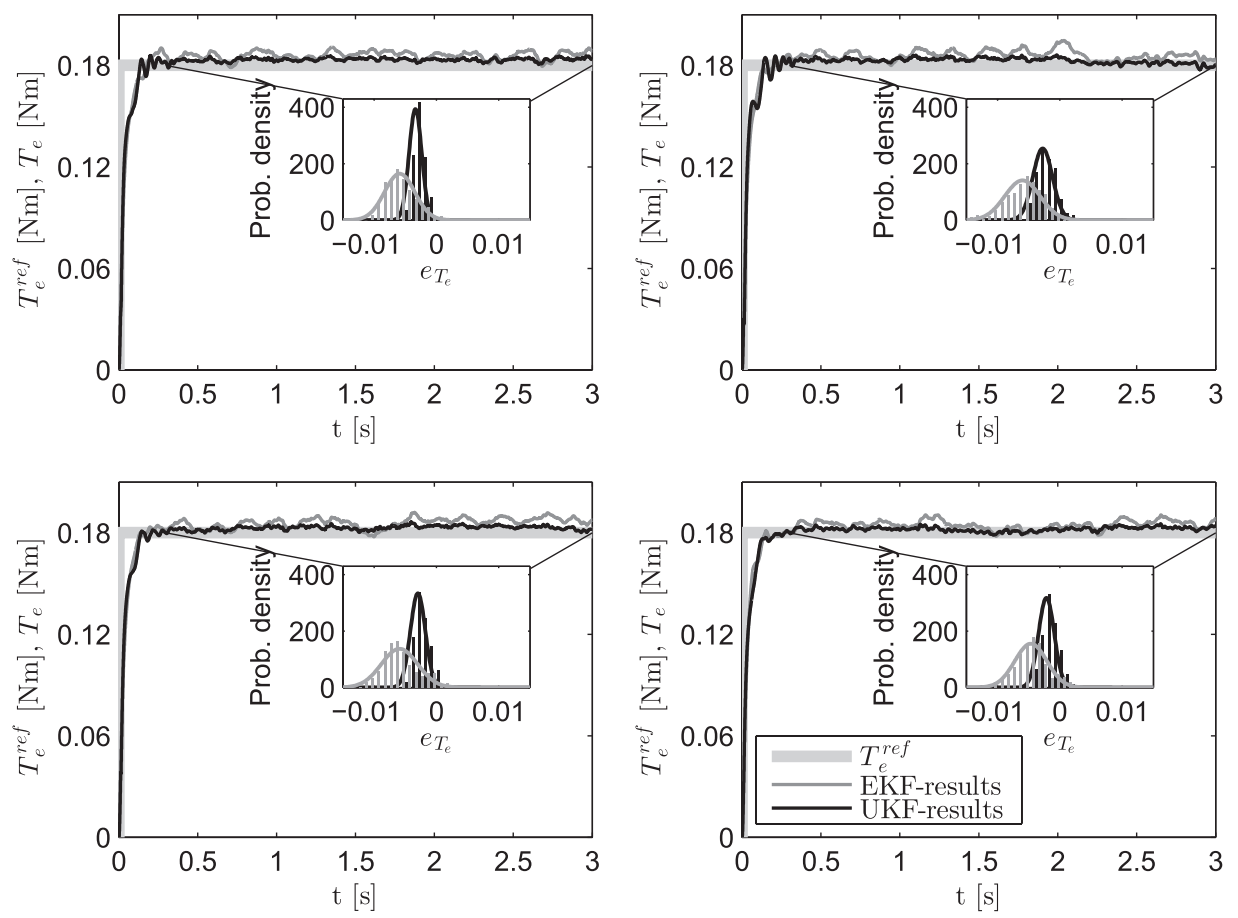

Fig. 7. Torque control performance besides different realisations of $W_{n}(t)$

Table 1. $\operatorname{Var}\left(e_{T_{e}}\right), t>0.3 \mathrm{~s}$ besides different realisations of $W_{n}(t)$

\begin{tabular}{|c|c|c|c|c|}
\hline Realisations & 1 & 2 & 3 & 4 \\
\hline $\operatorname{Var}\left(e_{T_{e}}\right)$ with EKF & $5.8760 \cdot 10^{-6}$ & $8.0138 \cdot 10^{-6}$ & $8.4543 \cdot 10^{-6}$ & $6.5985 \cdot 10^{-6}$ \\
\hline $\operatorname{Var}\left(e_{T_{e}}\right)$ with UKF & $1.0254 \cdot 10^{-6}$ & $2.4629 \cdot 10^{-6}$ & $1.4295 \cdot 10^{-6}$ & $1.5731 \cdot 10^{-6}$ \\
\hline
\end{tabular}

\section{Conclusion}

In this paper, an estimator-based speed sensorless DRFOC algorithm has been introduced for induction machines. The state estimator of the applied control method is based on a seventh-order non-linear state space model of the machine, which provides a detailed description of it. Among many other state variables, the estimator estimates the load torque as well. By using this feature, the speed sensorless control algorithm can provide satisfactory control performance besides varying load.

In order to estimate the state variables, two different estimator algorithms are applied and discussed, namely, the conventional EKF and the more advanced UKF. For the comparison of control performances of the EKF- and the UKF-based control methods, various simulations and experiments have been implemented.

Step response simulations and experiments show that the UKF-based control algorithm provides similar dynamic performance as the EKF-based one. Besides stochastically varying load torque disturbance, however, the performance advantage of the UKF-based algorithm becomes apparent. In that case, the performance of the EKF-based algorithm degrades as it estimates the load and keeps the torque reference with higher errors than the UKF. 


\section{References}

Akin, B., Orguner, U., Ersak, A. and Ehsani, M. (2006). Simple Derivative-Free Nonlinear State Observer for Sensorless AC Drives. IEEE/ASME Transactions on Mechatronics, 11(5), pp. 634-643.

Amezquita-Brooks, L., Liceaga-Castro, E., LiceagaCastro, J. and Ugalde-Loo, C. E. (2015). FluxTorque Cross-Coupling Analysis of FOC Schemes: Novel Perturbation Rejection Characteristics. ISA Transactions, 58, pp. 446-461.

Auger, F., Hilairet, M., Guerrero, J. M., Monmasson, E., Orłowska-Kowalska, T. and Katsura, S. (2013). Industrial Applications of the Kalman Filter: A Review. IEEE Transactions on Industrial Electronics, 60(12), pp. 5458-5471.

Biswas, S. K., Qiao, L. and Dempster, A. G. (2017). A Novel A Priori State Computation Strategy for the Unscented Kalman Filter to Improve Computational Efficiency. IEEE Transactions on Automatic Control, 64(4), pp. 1852-1864.

Casadei, D., Profumo, F., Serra, G. and Tani, A. (2002). FOC and DTC: Two Viable Schemes for Induction Motors Torque Control. IEEE Transactions on Power Electronics, 17(5), pp. 779-787.

De Pelegrin, J., Torrico, C. R. C. and Carati, E. G. (2016). A Model-Based Suboptimal Control to Improve Induction Motor Efficiency. Journal of Control, Automation and Electrical Systems, 27(1), pp. 69-81.

Eisenberg, B. and Sullivan, R. (2008). Why is the Sum of Independent Normal Random Variables Normal? Mathematics Magazine, 81(5), pp. 362-366.

Fodor, D. and Tóth, R. (2004). Speed sensorless linear parameter variant $\mathrm{H}_{\infty}$ control of the induction motor. In: 2004 43rd IEEE Conference on Decision and Control (CDC) (IEEE Cat. No.04CH37601), Vol. 4. Nassau (Bahamas): IEEE, pp. 4435-4440.

Grafarend, E. W. (2006). Linear and Nonlinear Models: Fixed Effects, Random Effects, and Mixed Models. Berlin/New York: Walter de Gruyter.

Holtz, J. (2006). Sensorless Control of Induction Machines - With or Without Signal Injection. IEEE Transactions on Industrial Electronics, 53(1), pp. 7-30.

Horváth, K. and Kuslits, M. (2017). Speed sensorless field oriented control of induction machines using unscented Kalman filter. In: 2017 International Conference on Optimization of Electrical and Electronic Equipment (OPTIM) 2017 International Aegean Conference on Electrical Machines and Power Electronics (ACEMP). Brasov: IEEE, pp. 523-528.
Jafarzadeh, S., Lascu, C. and Fadali, M. S. (2013). Square Root Unscented Kalman Filters for State Estimation of Induction Motor Drives. IEEE Transactions on Industry Applications, 49(1), pp. 92-99.

Jafarzadeh, S., Lascu, C. and Fadali, M. S. (2012). State Estimation of Induction Motor Drives Using the Unscented Kalman filter. IEEE Transactions on Industrial Electronics, 59(11), pp. 4207-4216.

Julier, S. J. and Uhlmann, J. K. (1997). A New Extension of the Kalman Filter to Nonlinear Systems. In: Proceedings of AeroSense: The 11th International Symposium on Aerospace/Defense Sensing, Simulations and Controls, Orlando.

Julier, S. J. and Uhlmann, J. K. (2004). Unscented Filtering and Nonlinear Estimation. Proceedings of the IEEE, 92(3), pp. 401-422.

Julier, S. J., Uhlmann, J. K. and Durrant-Whyte, H. F. (2000). A New Method for the Nonlinear Transformation of Means and Covariances in Filters and Estimators. IEEE Transactions on Automatic Control, 45(3), pp. 477-482.

Kalman, R. E. (1960). A New Approach to Linear Filtering and Prediction Problems. Journal of Basic Engineering, 82(1), pp. 35-45.

Kim, Y.-R., Sul, S.-K. and Park, M.-H. (1994). Speed Sensorless Vector Control of Induction Motor Using Extended Kalman Filter. IEEE Transactions on Industry Applications, 30(5), pp. 1125-1133.

Kumar, S., Prakash, J. and Kanagasabapathy, P. (2011). A Critical Evaluation and Experimental Verification of Extended Kalman Filter, Unscented Kalman Filter and Neural State Filter for State Estimation of Three Phase Induction Motor. Applied Soft Computing, 11(3), pp. 3199-3208.

Lalley, S. (2001). Stochastic Calculus and Finance I, Lecture 5: Brownian Motion. Lecture Notes, The University of Chicago.

Leite, A. V., Araujo, R. E. and Freitas, D. (2004). Full and reduced order extended Kalman filter for speed estimation in induction motor drives: A comparative study. In: 2004 IEEE 35th Annual Power Electronics Specialists Conference (IEEE Cat. No.04CH37551) Vol. 3. Aachen (Germany): IEEE, pp. 2293-2299.

Lešić, V., Vašak, M., Stojičić, G., Perić, N., Joksimović, G. and Wolbank, T. M. (2012). State and parameter estimation for field-oriented control of induction machine based on unscented Kalman filter. In: International Symposium on Power Electronics Power Electronics, Electrical Drives, Automation and Motion. Sorrento: IEEE, pp. 409-414. 
Liu, K.-Z., Yokoo, M., Kondo, K. and Zanma, T. (2015). New Adaptive Vector Control Methods for Induction Motors with Simpler Structure and Better Performance. Control Theory and Technology, 13(2), pp. 173-183.

Loeve, M. (2012). Probability Theory I. Graduate Texts in Mathematics. New York: Springer-Verlag.

Orłowska-Kowalska, T. and Dybkowski, M. (2016). Industrial Drive Systems. Current State and Development Trends. Power Electronics and Drives, 1(1), pp. 5-25.

Rigatos, G. and Siano, P. (2012). Sensorless nonlinear control of induction motors using unscented Kalman filtering. In: IECON 2012 - 38th Annual Conference on IEEE Industrial Electronics Society. Montreal: IEEE, pp. 4654-4659.

Šlapák, V., Kyslan, K. and Ďurovský, F. (2016a). Position Controller for PMSM Based on Finite Control Set Model Predictive Control. Elektronika ir Elektrotechnika, 22(6), pp. 17-21.

Šlapák, V., Kyslan, K., Lacko, M., Fedák, V. and Duurovský, F. (2016b). Finite Control Set Model Predictive Speed Control of a DC Motor. Mathematical Problems in Engineering, pp. 1-10.
Vantsevich, V. V. and Blundell, M. V. (2015). Advanced Autonomous Vehicle Design for Severe Environments. Amsterdam: IOS Press.

Yildiz, R., Barut, M. and Zerdali, E. (2016). Speedsensorless induction motor drive with unscented Kalman filter including the estimations of load torque and rotor resistance. In: IECON 2016 42nd Annual Conference of the IEEE Industrial Electronics Society. Florence: IEEE, pp. 29462950.

Yildiz, R., Barut, M., Zerdali, E., Inan, R. and Demir, R. (2017). Load torque and stator resistance estimations with unscented Kalman filter for speed-sensorless control of induction motors. In: 2017 International Conference on Optimization of Electrical and Electronic Equipment (OPTIM) 2017 International Aegean Conference on Electrical Machines and Power Electronics (ACEMP). Brasov: IEEE, pp. 456-461.

Zerdali, E. and Barut, M. (2017). The Comparisons of Optimized Extended Kalman Filters for SpeedSensorless Control of Induction Motors. IEEE Transactions on Industrial Electronics, 64(6), pp. 4340-4351. 